\title{
Some Results on a Generalized Archimedean Family of Copulas
}

\author{
Ali Dolati* and Mojdeh Karbasian \\ Yazd University
}

\begin{abstract}
Durante et al. (2007) introduced a class of bivariate copulas depending on two generators which generalizes some known families such as the Archimedean copulas. In this paper we provide some result on properties of this family when the generators are certain univariate survival functions.
\end{abstract}

Keywords. Copula; Archimedean copula; dependence ordering; survival function.

MSC 2010: 62H20, 60E15.

\section{Introduction}

The construction of distributions with given marginals has been a subject to various lines of statistical research. In view of Sklar's Theorem (Sklar, 1959) this problem can be reduced to the construction of a copula. Nelsen (2006) summarizes different methods of constructing copulas. Among these methods, constructing principles for copulas based on certain univariate functions have gained in importance: see, for instance Amblard and Girard (2002), Rodríguez-Lallena and Úbeda-Flores (2004), Morillas (2005), Fischer and Klein (2006), Durante (2007) and Durante et al. (2007). Most frequently, Archimedean copulas are used which constructed by composition of a specific generator and its pseudo inverse: see, for instance Genest and Rivest (1993) and Avérous and Dortet-Bernadet (2004). Durante, et al. (2007) introduced a class of bivariate copulas, depending on two generators which

\footnotetext{
* Corresponding author
} 
generalizes the well-known Archimedean family. The contribution of this paper is to study this generalized family of copulas, when the generators are certain univariate survival functions in the same spirit as Avérous and Dortet-Bernadet (2004). Since one can find a large and diverse collection of univariate survival functions in the literature, choosing the survival functions as the generators can be useful in building new parametric families of Archimedean copulas in a simple manner. The paper is organized as follows. We recall some preliminary results on copulas and stochastic orderings that we need in the sequel in Section 2. In Section 3, we discussed the proposed model and provide a condition for Archimedeanity. In Section 4 we study special cases and provide several new and already-existent extensions of the Archimedean copulas. Section 5 devoted to the dependence orderings of the model in terms of the variability and aging orderings of the generators. Finally we conclude the paper in Section 6 .

\section{Preliminaries}

We recall that a (bivariate) copula is a function $C:[0,1] \times[0,1] \rightarrow[0,1]$ such that: (i) $C(u, 0)=C(0, u)=0$ and $C(u, 1)=C(1, u)=u$ for every $u \in[0,1]$; (ii) $C$ is 2-increasing, viz., for every $u_{1} \leqslant u_{2}$ and $v_{1} \leqslant v_{2}$, $V_{C}\left(\left[u_{1}, u_{2}\right] \times\left[v_{1}, v_{2}\right]\right)=C\left(u_{1}, v_{1}\right)+C\left(u_{2}, v_{2}\right)-C\left(u_{1}, v_{2}\right)-C\left(u_{2}, v_{1}\right) \geqslant 0$. Every copula is bounded below by $W(u, v)=\max \{u+v-1,0\}$ and above by $M(u, v)=\min (u, v)$, the so-called Fréchet-Hoeffding bounds. Moreover, the copula associated with the joint distribution of two independent random variables is given by $\Pi(u, v)=u v$. A copula is Archimedean if there exists a convex and decreasing function $\phi:[0,1] \rightarrow[0, \infty]$ with $\phi(1)=0$, such that $C(u, v)=\phi^{-1}\{\phi(u)+\phi(v)\}$ with the convention $\phi^{-1}(u)=0$ if $u>\phi(0)$. The function $\phi$, is called an additive generator of the copula $C$ (Nelsen, 2006). Throughout the paper, increasing means nondecreasing and decreasing means nonincreasing. For $i=1,2$, let us denote by $S_{i}(t)=P\left(X_{i}>t\right)$ the survival function of the continuous and non-negative random variable $X_{i}$. Let also $S_{1}(0)=S_{2}(0)=1$. The results to be described below depend on the following well-known notions of aging notions (Barlow and Proschan, 1975): Increasing Failure Rate (IFR) and New Better than Used (NBU). These concepts are defined as follows: $S_{2} \prec_{\text {IFR }} S_{1}$, if and only if $S_{1}^{-1} \circ S_{2}$ is convex; $S_{2} \prec_{\mathrm{NBU}} S_{1}$, if and only if $S_{1}^{-1} \circ S_{2}$ is superadditive, i.e., $S_{1}^{-1} \circ S_{2}(x+y) \geqslant S_{1}^{-1} \circ S_{2}(x)+S_{1}^{-1} \circ S_{2}(y)$, where $S^{-1}(u), u \in(0,1)$, denote the right-continuous inverse of $S$, defined by $S^{-1}(u)=\sup \{x: S(x) \geqslant u\}$. 
Let $K(t)=e^{-t}, t \geqslant 0$, be the exponential survival function. The survival function $S$ is IFR if $S \prec_{\mathrm{IFR}} K$ and survival function $S$ is NBU if $S \prec_{\mathrm{NBU}} K$. The corresponding negative concepts of Decreasing Failure Rare (DFR) and New Worse than Used (NWU) can be defined mutatis mutandis. In the sequel, we shall also need some notions of stochastic orderings. We denote by $S_{2} \prec_{\text {st }} S_{1}$ the usual stochastic dominance $S_{1}(t) \geqslant S_{2}(t)$ for all $t \geqslant 0$, or equivalently, $S_{1}^{-1}(u) \geqslant S_{2}^{-1}(u)$ for all $u \in(0,1)$. The classical dispersive ordering between univariate distributions, whose definition is recalled below, also plays a role in the sequel. A survival function $S_{2}$ is said to be dispersed with respect to another survival function $S_{1}$ (denoted by, $S_{2} \prec_{\text {Disp }} S_{1}$ ) if, and only if $S_{2}^{-1}(u)-S_{1}^{-1}(u)$ is increasing in $u \in(0,1)$. Equivalently, one must have $S_{2}\left\{S_{2}^{-1}(\alpha)+c\right\} \leqslant S_{1}\left\{S_{1}^{-1}(\alpha)+c\right\}$, for all $\alpha \in(0,1)$ and $c>0$. Notice that if $S_{1}(0)=S_{2}(0)=1$, then $S_{2} \prec_{\text {Disp }} S_{1}$ implies $S_{2} \prec_{s t} S_{1}$; (see Shaked and Shanthikumar, 2007 for detail). By analogy with the univariate notion of stochastic dominance, copula $C_{1}$ is said to be more dependent than $C_{2}$ in the positive quadrant dependence (PQD) ordering, denoted by $C_{2} \prec C_{1}$, if and only if, $C_{2}(u, v) \leqslant C_{1}(u, v)$ for all $u, v \in(0,1)$. A copula $C$ is said to be PQD (resp, NQD) if $C \succ \Pi$ (resp, $C \prec \Pi$ ). A stronger dependence ordering is left-tail decreasingness LTD is defined in terms of the conditional distributions $C_{i, u}^{L}(v)=\frac{1}{u} C_{i}(u, v)=P\left(V_{i} \leqslant v \mid U_{i} \leqslant u\right)$, and their (right continuous) inverses $\left(C_{i, u}^{L}\right)^{-1}, i=1,2$. The copula $C_{1}$ is said to be less LTD than another copula $C_{2}$, denoted by $C_{1} \prec_{\mathrm{LTD}} C_{2}$, if and only if $C_{2, u_{2}}^{L} \circ\left(C_{2, u_{1}}^{L}\right)^{-1}(v) \leqslant C_{1, u_{2}}^{L} \circ\left(C_{1, u_{1}}^{L}\right)^{-1}(v)$ for all $0<u_{1} \leqslant u_{2}<1$ and $v \in(0,1)$. The LTD ordering is stronger than PQD in the sense that $C_{1} \prec_{\text {LTD }} C_{2}$ implies that $C_{1} \prec C_{2}$. A copula $C$ is LTD if $\Pi \prec_{\text {LTD }} C$, that is, $C_{u}^{L}(v)$ is decreasing in $u$ for all $v \in(0,1)$ (see Avérous and Dortet-Bernadet, 2004 for detail).

\section{The Generalized Family}

Durante et al. (2007) introduced a class of bivariate copulas, depending on two univariate functions which generalizes the Archimedean family, of the form

$$
C(u, v)=\phi^{-1}\{\phi(u \wedge v)+\psi(u \vee v)\}, \quad u, v \in[0,1]
$$

where $x \wedge y=\min (x, y), x \vee y=\max (x, y) . \phi$ and $\psi$ are two continuous functions on $[0,1] ; \phi$ is strictly decreasing and $\psi$ is decreasing with $\psi(1)=0$. They proved that if $\phi$ is convex and $\psi-\phi$ is increasing, then (1) is a copula. 
When $\phi=\psi$ the copula (1) reduces to the class of Archimedean copulas.

For each generator $\phi$ of an Archimedean copula the function $S_{\phi}(t)=$ $\phi^{-1}(t)$ is a convex survival function. In particular, the exponential survival function $S_{\phi_{0}}(t)=e^{-t}$, is associated with the generator $\phi_{0}(t)=-\ln (t)$ of the product copula $\Pi$. Motivated by this fact, let $\Gamma$ be the class of all continuous and strictly decreasing survival functions $S:[0, \infty] \rightarrow[0,1]$, with $S(0)=1$ and $S(\infty)=0$; and consider the function $C:[0,1] \times[0,1] \rightarrow[0,1]$, defined by

$$
C(u, v)=S_{1}\left\{S_{1}^{-1}(u \wedge v)+S_{2}^{-1}(u \vee v)\right\}, \quad u, v \in[0,1]
$$

where $S_{1}, S_{2}, \in \Gamma$. The following proposition which is a special case of Theorem 3.1 in Durante et al. (2007) provides under which conditions on $S_{1}$ and $S_{2}$, the function $C$, defined by (2) is a copula.

Proposition 1. Let $S_{1}$ and $S_{2}$ be two survival functions in $\Gamma$. If $S_{1}$ is convex and $S_{2} \prec_{\text {Disp }} S_{1}$ then the function $C$ defined by (2) is a copula.

Proof. Since for two survival function $S_{1}$ and $S_{2}, S_{1} \prec_{\text {Disp }} S_{2}$ if and only if $S_{2}^{-1}(x)-S_{1}^{-1}(x)$ is increasing in $x$, the proof is immediate from Theorem 3.1 in Durante et al. (2007), by replacing $\phi_{i}(x)=S_{i}^{-1}(x), i=1,2$.

We call a generator a pair of survival functions $\left(S_{1}, S_{2}\right)$ that generates a copula of the form (2).

When the generator $\left(S_{1}, S_{2}\right)$ satisfies $S_{1}=S_{2}$, the copulas of type (2) are Archimedean. When $S_{1} \neq S_{2}$, the copula $C$ may be Archimedean; see, e.g., Section 4 in Durante et al. (2007). In the following we shall investigate under which conditions on $S_{1}$ and $S_{2}$, the copula $C$, defined by (2) is Archimedean, providing a response to a question posed in Section 4 of Durante et al. (2007).

Proposition 2. A copula $C$ of type (2) is Archimedean if and only if $S_{1}(x)=S_{2}(x+c)$ for some constant $c$ and all $x>0$.

Proof. If $S_{1}(x)=S_{2}(x+c)$, then it is easy to see that $C(u, v)=S_{2}\left\{S_{2}^{-1}(u)+\right.$ $\left.S_{2}^{-1}(v)\right\}$, which is an Archimedean copula with the generator $\phi(x)=S_{2}^{-1}(x)$. Now we prove that the Archimedeanity of a copula of the form (2) leads to the condition $S_{1}(x)=S_{2}(x+c)$. Let $\delta_{C}(t)=C(t, t), t \in(0,1)$, be the diagonal section of $C$. Since $S_{1}$ and $S_{2}$ are decreasing and $S_{2}(0)=1$, one has that $\delta_{C}(t)<t$, for all $t \in(0,1)$. This property and associativity of $C$, 
i.e., $C(C(u, v), w)=C(u, C(v, w))$, for all $u, v, w \in[0,1]$, characterize the Archimedean copulas. To check the associativity of $C$, assume, first, that $(u, v),(v, w) \in\left\{(x, y) \in[0,1]^{2}: x \leqslant y\right\}$. Then

$$
\begin{aligned}
& C(C(u, v), w)=S_{1}\left\{S_{1}^{-1}(u)+S_{2}^{-1}(v)+S_{2}^{-1}(w)\right\}, \text { and } \\
& C(u, C(v, w))=S_{1}\left\{S_{1}^{-1}(u)+S_{2}^{-1}\left[S_{1}\left(S_{1}^{-1}(v)+S_{2}^{-1}(w)\right)\right]\right\} .
\end{aligned}
$$

We have $C(C(u, v), w)=C(u, C(v, w))$ if and only if $S_{1}\left\{S_{1}^{-1}(v)+S_{2}^{-1}(w)\right\}=$ $S_{2}\left\{S_{2}^{-1}(v)+S_{2}^{-1}(w)\right\}$, for all $v \leqslant w$. Taking $S_{1}^{-1}(v)=x, S_{2}^{-1}(w)=y$, we must have the functional equation $g(x+y)=g(x)+y$ where $g(t)=S_{2}^{-1} \circ S_{1}(t)$. The general solution is $g(t)=t+c$; see Aczél (1966). Thus we need to have $S_{1}(t)=S_{2}(t+c)$, for some $c$ and all $t \geqslant 0$. Since the Archimedean copula $C$ is symmetric, i.e., $C(u, v)=C(v, u)$, the same argument holds for the associativity of $C$, in the case $(u, v),(v, w) \in\left\{(x, y) \in[0,1]^{2}: x>y\right\}$, which completes the proof.

Example 1. Consider the Pareto survival function given by $S_{1}(x)=$ $\{1+(x-\theta)\}^{-\frac{1}{\alpha}}$ with $x \geqslant \theta$ and $\alpha>0$ and let $S_{2}(x)=S_{1}(x+\theta)$. Then the resulting copula (2) is given by

$$
C(u, v)=\left\{u^{-\alpha}+v^{-\alpha}-1\right\}^{-\frac{1}{\alpha}}
$$

which is a member of the well-known Clayton's family of Archimedean copulas (Nelsen, 2006) with the generator $\phi(x)=x^{-\alpha}-1$.

\section{Special Cases and New Examples}

Notice that, if $\left(S_{1}, S_{2}\right)$ is a pair of survival functions that generates a copula of the form (2), then for any $\alpha \in(0,1]$, the pair $\left(S_{1}^{\frac{1}{\alpha}}, S_{2}^{\frac{1}{\alpha}}\right)$ generates a copula of the form (2). Moreover, another generator is given by the pair $\left(G_{1}, G_{2}\right)$, where $G_{i}(t)=S_{i}\left(t^{\frac{1}{\alpha}}\right)$, for every $\alpha \geqslant 1$; see also Remark 2 in Durante et al. (2007). For $i=1,2$, let $S_{i}(t)=S\left(\frac{t}{\theta_{i}}\right)$, with $t$ and $\theta>0$, for some convex and decreasing survival function $S$. Then, $S_{i}^{-1}(u)=\theta_{i} S^{-1}(u), u \in[0,1]$. It is easy to see that $S_{2} \prec$ Disp $S_{1}$ if, and only if $\theta_{1} \geqslant \theta_{2}$. The corresponding copula of the form (2) is given by

$$
C_{\alpha}(u, v)=S\left\{S^{-1}(u \wedge v)+\alpha S^{-1}(u \vee v)\right\},
$$


where $0<\alpha=\theta_{2} / \theta_{1} \leqslant 1$. Since the family of copulas defined by (3) depends only to one generator, it may have some independent interest. Note that $\lim _{\alpha \rightarrow 0} C_{\alpha}(u, v)=\min (u, v)$, and the case $\alpha=1$ reduces to the Archimedean copula

$$
C_{1}(u, v)=S\left\{S^{-1}(u)+S^{-1}(v)\right\} .
$$

When $S(t)=e^{-t}, t \geqslant 0$, the generated copula by (3) is $C_{\alpha}(u, v)=\min \left\{u v^{\alpha}\right.$, $\left.u^{\alpha} v\right\}$ which is a member of the Cuadras-Agué family of copulas; see also Example 4.1 in Durante et al. (2007).

Example 2. Consider the survival function given by $S(t)=\frac{1}{1+t}, t \geqslant 0$. Then (3) yields a new two parameter family of copulas of the form

$$
C_{\alpha}(u, v)=\frac{u v}{\max (u, v)+\alpha(\min (u, v)-u v)} .
$$

Consider the generator $(K, S)$, where $K$ is the survival function of the ordinary exponential distribution given by $K(t)=e^{-t}, t \geqslant 0$ and $S$ is a survival function in $\Gamma$. Then, $S \prec_{\text {Disp }} K$, if and only if $\frac{e^{-t}}{S(t)}$ is increasing in $t \geqslant 0$. If $S$ has a density, by differentiating, this condition can be recast as the condition that $-\frac{d}{d t} \ln S(t) \geqslant 1$. The corresponding copula of type (2) is given by

$$
C(u, v)=\min (u, v) \exp \left\{-S^{-1}(u \vee v)\right\} .
$$

The class of copulas of the form $C(u, v)=\min (u, v) f(u \vee v)$, where $f$ is a suitable function from $[0,1]$ to $[0,1]$, is studied in Durante $(2007)$.

Example 3. Let $S(t)=\exp \left\{\theta\left(1-e^{t}\right)\right\} t \geqslant 0$. It is easy to see that $S$ satisfies $-\frac{d}{d t} \ln S(t) \geqslant 1$, for $\theta \geqslant 1$. Then the copula generated by (4) is given by

$$
C_{\theta}(u, v)=\frac{\theta \min (u, v)}{\theta-\ln (u \vee v)}
$$

Notice that $C_{\infty}=M$.

Example 4. For $i=1,2$, let $S_{i}(t)=\frac{1-\theta_{i}}{e^{t}-\theta_{i}}, t \geqslant 0, \theta_{i} \in[0,1)$. This survival functions has investigated in Marshall and Olkin (1979). Since $\ln S_{1}$ is convex 
for $\theta \leqslant 1$, then $S_{1}$ is a convex function for $\theta \leqslant 1$. We also have that, $S_{2} \prec$ Disp $S_{1}$, if and only if $\theta_{1} \leqslant \theta_{2}$. The generated copula of type (2) is given by

$$
C_{\theta_{1}, \theta_{2}}(u, v)=\frac{\left(1-\theta_{1}\right) u v}{\left(1-\theta_{1} \max (1-u, 1-v)\right)\left(1-\theta_{2} \min (1-u, 1-v)\right)-\theta_{1} u v} .
$$

Notice that the case, $\theta_{1}=\theta_{2}=\theta$, reduces to the Ali-Mikhail-Haq family of copulas (Nelsen, 2006) given by $C_{\theta}(u, v)=u v /\{1-\theta(1-u)(1-v)\}$.

Example 5. For $i=1,2$, let $S_{i}(t)=\left(\frac{1}{1+\theta_{i} t}\right)^{\frac{1}{\theta_{i}}}, t \geqslant 0, \theta_{i}>0$. It is easy to see that $S_{1}$ and $S_{2}$ satisfy the conditions in Proposition 1 , if $\theta_{1} \geqslant \theta_{2}$. The generated copula of type (2) is given by

$$
C_{\theta_{1}, \theta_{2}}(u, v)=\left\{(u \wedge v)^{-\theta_{1}}+\frac{\theta_{1}}{\theta_{2}}(u \vee v)^{-\theta_{2}}-1\right\}^{\frac{-1}{\theta_{1}}} .
$$

The case $\theta_{1}=\theta_{2}=\theta$, reduces to the Clayton's family of copulas.

Example 6. Let $S_{1}(t)=\frac{1}{1+t^{\frac{1}{\alpha}}}$ and $S_{2}(t)=e^{-t^{\frac{1}{\alpha}}}, t \geqslant 0$, then $S_{1}$ is convex and $S_{2} \prec$ Disp $S_{1}$, whenever $\alpha \geqslant 1$. The generated copula $C_{\alpha}$ is given by

$$
C_{\alpha}(u, v)=\left\{(u \wedge v)^{-\alpha}+\ln (u \vee v)^{-\alpha}\right\}^{\frac{-1}{\alpha}} .
$$

\section{Dependence Orderings and Measures}

In the following, we study the dependence orderings of the copulas of the form (2). These copulas, as we will see, has this nice property that: the degree of dependence increases as the variability of the generator survival functions decreases. The part (i) of the following Proposition is comparable with the Proposition 5.1 in Durante et al. (2007). Our findings extend the results presented in Section 5 of Durante et al. (2007).

Proposition 3. Let $C$ and $D$ be two copulas of type (2), generated by the pairs $\left(S_{1}, S_{2}\right)$ and $\left(K_{1}, K_{2}\right)$ respectively. 
(i) If $K_{1} \prec_{\mathrm{NBU}} S_{1}$ and $S_{1}^{-1} \circ K_{1}(u) \geqslant S_{2}^{-1} \circ K_{2}(u)$ for all $u \in(0,1)$ then $D \prec C$;

(ii) If $K_{1} \prec_{\mathrm{IFR}} S_{1}$ and $S_{2}^{-1} \circ K_{2}(x)-S_{1}^{-1} \circ K_{1}(x)$ is decreasing in $x$ then $D \prec_{\text {LTD }} C$.

Proof. Let $u$ and $v$ be in $[0,1]$ and assume that $u \leqslant v$. Then, $D \prec C$, if and only if $K_{1}\left\{K_{1}^{-1}(u)+K_{2}^{-1}(v)\right\} \leqslant S_{1}\left\{S_{1}^{-1}(u)+S_{2}^{-1}(v)\right\}$. Let $K_{1}^{-1}(u)=a$ and $K_{2}^{-1}(v)=b$ and apply the transformation $S_{1}^{-1}$ on both sides of this inequality. Then, we need to have $S_{1}^{-1} \circ K_{1}\{a+b\} \geqslant S_{1}^{-1} \circ K_{1}(a)+S_{2}^{-1} \circ K_{2}(b)$, for all $a \geqslant b$. Now, under the assumptions $K_{1} \prec_{\mathrm{NBU}} S_{1}$ (i.e., $S_{1}^{-1} \circ K_{1}$ is superadditive) and $S_{1}^{-1} \circ K_{1}(u) \geqslant S_{2}^{-1} \circ K_{2}(u)$, we have that

$$
\begin{aligned}
S_{1}^{-1} \circ K_{1}\{a+b\} & \geqslant S_{1}^{-1} \circ K_{1}(a)+S_{1}^{-1} \circ K_{1}(b) \\
& \geqslant S_{1}^{-1} \circ K_{1}(a)+S_{2}^{-1} \circ K_{2}(b),
\end{aligned}
$$

whence the result. If $u>v$, then proof can be completed using the same arguments. For part (ii), by definition, $D \prec_{\text {LTD }} C$ if, and only if, $C_{u_{2}}^{L} \circ$ $\left(C_{u_{1}}^{L}\right)^{-1}(v) \leqslant D_{u_{2}}^{L} \circ\left(D_{u_{1}}^{L}\right)^{-1}(v)$, for all $0 \leqslant u_{1} \leqslant u_{2} \leqslant 1$ and all $v \in(0,1)$. First assume that $\left(u_{i}, v\right) \in\{(u, v): u \leqslant v\}, i=1,2$ and $0 \leqslant u_{1} \leqslant u_{2} \leqslant 1$. Then $D \prec_{\text {LTD }} C$ if and only if,

$S_{1}\left\{S_{1}^{-1}\left(u_{2}\right)+S_{1}^{-1}\left(u_{1} v\right)-S_{1}^{-1}\left(u_{1}\right)\right\} \leqslant K_{1}\left\{K_{1}^{-1}\left(u_{2}\right)+K_{1}^{-1}\left(u_{1} v\right)-K_{1}^{-1}\left(u_{1}\right)\right\}$.

Applying the transformation $S_{1}^{-1}$ on both sides of this inequality, we see that $S_{1}^{-1} \circ K_{1}\left\{K_{1}^{-1}\left(u_{2}\right)+K_{1}^{-1}\left(u_{1} v\right)-K_{1}^{-1}\left(u_{1}\right)\right\} \leqslant S_{1}^{-1}\left(u_{2}\right)+S_{1}^{-1}\left(u_{1} v\right)-S_{1}^{-1}\left(u_{1}\right)$.

By taking $K_{1}^{-1}\left(u_{1}\right)=t, K_{1}^{-1}\left(u_{1} v\right)-K_{1}^{-1}\left(u_{1}\right)=h$ and $K_{1}^{-1}\left(u_{2}\right)=s$, the inequality (6) amounts to

$$
S_{1}^{-1} \circ K_{1}(s+h)-S_{1}^{-1} \circ K_{1}(s) \leqslant S_{1}^{-1} \circ K_{1}(t+h)-S_{1}^{-1} \circ K_{1}(t),
$$

for all $s \leqslant t$ and $h>0$. But inequality (7) characterizes the convexity of $S_{1}^{-1} \circ K_{1}(x)$ (see Marshal and Olkin, 1979) which follows from the assumption $K_{1} \prec_{\mathrm{IFR}} S_{1}$. Similarly, for the case $\left(u_{i}, v\right) \in\{(u, v): u>v\}, i=1,2$ and $0 \leqslant u_{1} \leqslant u_{2} \leqslant 1$, we have $D \prec_{\text {LTD }} C$ if and only if $S_{1}\left\{S_{1}^{-1}\left(u_{1} v\right)-S_{2}^{-1}\left(u_{1}\right)+S_{2}^{-1}\left(u_{2}\right)\right\} \leqslant K_{1}\left\{K_{1}^{-1}\left(u_{1} v\right)-K_{2}^{-1}\left(u_{1}\right)+K_{2}^{-1}\left(u_{2}\right)\right\}$. 
Again, by applying transformation $S_{1}^{-1}$ on both sides of this inequality, we obtain

$S_{1}^{-1} \circ K_{1}\left\{K_{1}^{-1}\left(u_{1} v\right)-K_{2}^{-1}\left(u_{1}\right)+K_{2}^{-1}\left(u_{2}\right)\right\} \leqslant S_{1}^{-1}\left(u_{1} v\right)-S_{2}^{-1}\left(u_{1}\right)+S_{2}^{-1}\left(u_{2}\right)$.

By taking $K_{2}^{-1}\left(u_{1}\right)=t, K_{1}^{-1}\left(u_{1} v\right)-K_{2}^{-1}\left(u_{1}\right)=h$ and $K_{2}^{-1}\left(u_{2}\right)=s$, the inequality (8) amounts to

$$
S_{1}^{-1} \circ K_{1}(s+h)-S_{2}^{-1} \circ K_{2}(s) \leqslant S_{1}^{-1} \circ K_{1}(t+h)-S_{2}^{-1} \circ K_{2}(t),
$$

for all $s \leqslant t$ and $h>0$. A sufficient condition for holding (9) is that, $S_{1}^{-1} \circ K_{1}(s)-S_{1}^{-1} \circ K_{1}(t) \leqslant S_{2}^{-1} \circ K_{2}(s)-S_{2}^{-1} \circ K_{2}(t)$, whenever $s \leqslant t$, or equivalently, $S_{2}^{-1} \circ K_{2}(x)-S_{1}^{-1} \circ K_{1}(x)$ is decreasing in $x$, which completes the proof.

The following result which is comparable with the Corollary 5.1 in Durante et al. (2007), provides conditions that ensures that a copula of type (2) has positive dependence.

Corollary 1. Let $C$ be a copulas of type (2), generated by the pair $\left(S_{1}, S_{2}\right)$.

(i) If $S_{1}$ is $N W U$ then $C$ is $P Q D$;

(ii) If $S_{1}$ is DFR then $C$ is LTD.

Proof. The copula $\Pi$ is generated by the pair $(K, K)$, where $K(t)=e^{-t}$, $t \geqslant 0$. Then, in view of Proposition $3, \Pi \prec C$, if and only if $S_{1}(a+b) \geqslant$ $S_{1}(a) S_{2}(b)$, for all $a, b \geqslant 0$. Since $S_{2} \prec$ Disp $S_{1}$, implies that $S_{1}(t) \geqslant S_{2}(t)$, for all $t \geqslant 0$, then the NWU property of $S_{1}$ yields the required result. To prove (ii), by definition, $C$ is LTD if and only if $\Pi \prec_{\text {LTD }} C$. Using Proposition ؟, we need to have $K \prec_{\text {DFR }} S_{1}$, i.e., $S_{1}$ is DFR and $S_{2}^{-1}\left(e^{-t}\right)-S_{1}^{-1}\left(e^{-t}\right)$ is decreasing in $t \geqslant 0$. But the later condition follows from the fact that $S_{2} \prec_{\text {Disp }} S_{1}$.

The following result which extends the result of Corollary 5.2 in Durante et al. (2007) provide two particular cases of dependence order between two copulas of type (2).

Corollary 2. Let $C$ and $D$ be two copulas of type (2), generated by the pairs $\left(S_{1}, S_{2}\right)$ and $\left(K_{1}, K_{2}\right)$, respectively. 
(i) If $K_{1}=S_{1}$, then $D \prec C$ if and only if $S_{2} \prec_{\mathrm{st}} K_{2}$ and $D \prec_{\mathrm{LTD}} C$ if and only if $S_{2} \prec_{\text {Disp }} K_{2}$;

(ii) If $K_{2}=S_{2}$, then $D \prec C$ if and only if $K_{1} \prec_{\text {Disp }} S_{1}$ and $D \prec_{\text {LTD }} C$ if and only if $K_{1} \prec_{\mathrm{IFR}} S_{1}$.

Example 7. The family of copulas $\left\{C_{\alpha}\right\}, \alpha \in(0,1]$, defined by (3) is negatively ordered, that is $C_{1} \prec C_{\alpha_{2}} \prec C_{\alpha_{1}} \prec M$, for $0<\alpha_{1} \leqslant \alpha_{2} \leqslant 1$. Thus $\left\{C_{\alpha}\right\}$ is more positively quadrant dependent than the Archimedean copula with the generator $\phi(t)=S^{-1}(t)$, for every $\alpha \in(0,1]$. Moreover, $\left\{C_{\alpha}\right\}$ is PQD if and only if $S$ has the NWU property.

Example 8. The family of copulas $\left\{C_{\theta}\right\}, \theta \geqslant 1$, defined by (5) is positively ordered, that is $C_{\theta_{1}} \prec C_{\theta_{2}}$, for $0<\theta_{1} \leqslant \theta_{2}$.

For statistical modelling, with each copula $C$ can associate - among others - two non-parametric measure of association, called Kendall's tau and medial correlation coefficient (Nelsen, 2006) which are respectively given by

$$
\begin{gathered}
\tau(C)=4 \int_{0}^{1} \int_{0}^{1} C(u, v) d C(u, v)-1, \\
\beta(C)=4 C\left(\frac{1}{2}, \frac{1}{2}\right)-1 .
\end{gathered}
$$

For the copula defined by $(2)$, generated by $\left(S_{1}, S_{2}\right)$, the measure $\beta(C)$ is given by

$$
\beta(C)=S\left\{M_{S_{1}}+\alpha M_{S_{2}}\right\},
$$

where $M_{S_{i}}=S^{-1}\left(\frac{1}{2}\right)$ is the median of the survival function $S_{i}, i=1,2$. The expression for the Kendall's tau associated with this family does not have a closed form. The following result provides an expression for the Kendall's tau associated with the class of copulas defined by (3).

Proposition 4. Let $C$ be the family of copulas defined by (3). Then

$$
\tau(C)=1-\frac{8 \alpha}{1+\alpha} \int_{0}^{1} t\left(S^{\prime}(t)\right)^{2} d t .
$$


Proof. Direct calculation using (10) gives the required result.

\section{Concluding Remarks}

We have discussed some results on a generalized family of copulas proposed by Durante et al. (2007), when the generators are certain univariate survival functions in the same spirit as the paper by Avérous and Dortet-Bernadet (2004). We provided a sufficient condition on the generators that this family is Archimedean and discussed dependence orderings of induced copulas in terms of the aging orderings of their corresponding generators. Since one can find a large and diverse collection of univariate survival functions in the literature, choosing the survival functions as the generators can be useful in building and studying new extensions for parametric families of Archimedean copulas in a simple manner.

\section{Acknowledgement}

The authors are very grateful to the reviewers for their valuable comments and suggestions which improve the presentation of this article.

\section{References}

Aczél, J. (1966). Lectures on Functional Equations and Their Applications, Academic Press, New York.

Amblard, C. and Girard, S. (2002). Symmetry and Dependence Properties within A Semi-Parametric Family of Bivariate Copulas. Journal of Nonparametric Statistics, 14, 715727.

Avérous, J. and Dortet-Bernadet, J.L. (2004). Dependence for Archimedean Copulas and Aging Properties of Their Generating functions. Sankhyā: The Indian Journal of Statistics, 66, 1-14.

Barlow, R.E. and Proschan, F. (1975). Statistical Theory of Reliability and Life Testing, Holt, Rinehart and Wiston, New York.

Durante, F. (2007). A New Class of Symmetric Bivariate Copulas. C. R. Acad. Sci. Paris, Ser. I, 344, 195-198.

Durante, F., Quesada-Molina, J. and Sempi, C. (2007). A Generalization of the Archimedean Class of Bivariate Copulas. Ann. Inst. Statist. Math. 59, 487-498.

J. Statist. Res. Iran 9 (2012): 147-158 
Fischer, M. and Klein, I. (2006). Constructing Generalized FGM Copulas by Means of Certain Univariate Distributions. Metrika, 65, 243-260.

Genest, C. and Rivest, L-P. (1993). Statistical Inference Procedures for Bivariate Archimedean Copulas. Journal of the American Statistical Association, 55, 698-707.

Marshall, A. and Olkin, I. (1979). Inequalities: Theory of Majorization and Its Application. Academic Press, New York.

Morillas, P.M. (2005). A Method to Obtain New Copulas from A Given One, Metrika, 61, 169-184.

Nelsen, R.B. (2006). An Introduction to Copulas. Springer, New York.

Rodríguez-Lallena, J.A. and Úbeda-Flores, M. (2004). A New Class of Bivariate Copulas. Statistics and Probability Letters, 66, 315-325.

Shaked, M. and Shanthikumar, G. (2007). Stochastic Orders. Springer, New York.

A. Sklar (1959). Fonctions De répartition á n Dimensions et Leurs Marges, Publ. Inst. Statist. Univ. Paris, 8, 229-231.

\author{
Ali Dolati \\ Department of Statistics, \\ College of Mathematics, \\ Yazd University, \\ Yazd, Iran. \\ email: adolati@yazd.ac.ir
}

\author{
Mojdeh Karbasian \\ Department of Statistics, \\ College of Mathematics, \\ Yazd University, \\ Yazd, Iran. \\ email: mkarbasiyan9@gmail.com
}

